\title{
EFFECTS OF POMEGRANATE EXTRACTS ON MMP-1, TIMP-1, MMP- 1/TIMP-1 RATIO, AND TYPE I COLLAGEN TO INHIBIT RIGHT VENTRICULAR FIBROSIS IN ANIMAL MODELS
}

\author{
Taufiq Hidayat ${ }^{1}$, I Ketut Alit Utamayasa ${ }^{1}$, Mahrus A. Rahman ${ }^{1 *}$, Teddy Ontoseno ${ }^{1}$ \\ ${ }^{1}$ Department of Child Health, Dr. Soetomo Hospital, Faculty of Medicine, Universitas Airlangga, Surabaya, \\ Indonesia \\ *mahrus_rahman@yahoo.com \\ https://doi.org/10.34302/crpjfst/2019.11.5.11 \\ Article history: \\ Received: \\ 9 March 2019 \\ Accepted: \\ 20 September 2019 \\ Keywords: \\ Pomegranate extracts; \\ Pulmonary arterial; \\ hypertension; \\ Right ventricular; \\ myocardial fibrosis.

\section{ABSTRACT} \\ Pulmonary arterial hypertension (PAH) may cause myocardial fibrosis. The \\ process involves collagenase, the metalloproteinase matrix (MMPs), and \\ tissue inhibitors of metalloproteinase (TIMPs). The accumulation of type I \\ collagen in the right ventricular heart muscle may result in right ventricular \\ fibrosis. Pomegranate extracts contain active ellagic acid ingredients with \\ anti-inflammatory, antiproliferative, antioxidant, and ACE inhibitory effects \\ on tissue fibrosis with no side effects. The aim of this study to evaluate the \\ effectiveness of pomegranate extracts on the fibrosis process due to PAH \\ especially on right ventricular myocardium by observing the number of cells \\ expressing MMP-1, TIMP-1, MMP-1/TIMP-1 ratio and type 1 collagen on \\ the right ventricular heart muscle of pulmonary arterial hypertension (PAH) \\ animal models. This study is an experimental laboratory research conducted \\ on Sprague-Dawley white mice (Rattus norvegicus). Subjects were grouped \\ as control and treatment groups that received CMC for two and four weeks, \\ respectively. Monocrotaline was injected to mice to induce pulmonary \\ arterial hypertension. MMP-1 expression of the right ventricular heart \\ muscle in the control group was lower $(p<0.001$ and $p<0.001)$. The number \\ of cells expressed in TIMP-1 was higher in the control group than the \\ observed pomegranate extracts for two weeks and four weeks $(\mathrm{p}<0.001)$. \\ The ratio of cell numbers expressing MMP-1/TIMP-1 was higher in the \\ pomegranate extract group than in the control group $(\mathrm{p}<0.001)$. The number \\ of cells expressing type I collagen in the pomegranate extract group was \\ lower than that of the control group $(\mathrm{p}<0.001)$. According to the result, \\ pomegranate extracts may inhibit the initial process of fibrogenesis.
}

\section{Introduction}

Pulmonary arterial hypertension (PAH) is defined as the mean pulmonary artery pressure (pPA) at rest exceeding $25 \mathrm{mmHg}$ (Wardle and Tulloh, 2012). PAH may cause myocardial fibrosis due to an imbalance of metalloproteinase-tissue inhibitors of metalloproteinases resulting in an accumulation of extracellular matrix (Jeffery et al., 2002; Mandal, 2014). The process involves collagenase, that is, the metalloproteinase matrix (MMPs) and tissue inhibitors of metalloproteinase (TIMPs) that affect the balance of type I collagen synthesis and degradation. The accumulation of type I collagen in the right ventricular heart muscle can cause right ventricular fibrosis. This results in a low cardiac output and activates the RAA system, triggering fibroblast proliferation and differentiation into collagen-secreting myofibroblasts.

Medication to prevent $\mathrm{PAH}$ progression while waiting for a definitive therapy is 
necessary to reduce morbidity and mortality (Rahman, 2016). Compared with calcium channel blockers, prostanoids, endothelin antagonists, and phosphodiesterase inhibitors (PDEs), each of which has different therapeutic points, pomegranate extracts have several advantages. Pomegranate extracts contain the active ingredient ellagic acid, which is rich in anti-inflammatory, antiproliferative, antioxidant, and ACE inhibitors that are beneficial for cardiovascular diseases and for the liver as an antifibrotic in mice models (Yuniarti et al., 2013; Haber et al., 2011). In addition to previous studies, pomegranates are said to have no side effects, so they are safe to be used as an alternative therapy (Vidal et al., 2003; Jurenka, 2008). However, there has been no research on pomegranates' effects on the right ventricular myocardial fibrosis process due to $\mathrm{PAH}$ caused by CHD with left-to-right shunting. The objective of this study is to examine the effectiveness of pomegranate extracts on fibrosis due to PAH especially on the right ventricular heart muscle by observing the number of cells expressing MMP-1, MMP1/TIMP-1 ratio, and type-1 collagen in the right ventricular hearts of PAH mice models.

\section{Materials and methods}

This study is an experimental research conducted in the Biochemistry Laboratory of the Faculty of Medicine, Universitas Airlangga. Ethical clearance was issued by the Animal Care and Use Committee, Veterinary Faculty, Universitas Airlangga, no: $758-\mathrm{KE}$, issued on August 10, 2017

\subsection{Sample}

This study used three-month-old SpragueDawley white mice (Rattus norvegicus) weighing 300-500 grams. Model mice were grouped as control and treatment groups. The control group was divided into two, which received $\mathrm{CMC} 0.3 \%$ treatment for two weeks (K1) and four weeks (E1), respectively. Treatment groups were divided into a group that received pomegranate extracts with $\mathrm{CMC} 0.3 \%$ for two weeks (K2) and another that received pomegranate extracts with $\mathrm{CMC}$ for $0.3 \%$ four weeks (E2).

\subsection{Monocoraltine and pomegranate extracts}

Monocrotaline (Chengdu Biopurify Phytochemicals, Chengdu, Sichuan, China) was subcutaneously injected to mice to induce PAH at $60 \mathrm{mg} / \mathrm{kg}$.

The materials in this research used extracts from all parts of pomegranate (Punica granatum $L$ ) in powder form and have been standardized, containing $40 \%$ ellagic acid, and produced by Xi'an Biof Bio-Technology Co., Ltd. (Room 11111, High-tech Venture Park, No. 69 Jinye Road, Gaoxin District of Xi'an, People's Republic of China).

\subsection{Blood analysis}

We performed a immunohistochemical analysis of monoclonal antibodies for MMP-1, TIMP-1, type 1 collagen, and MMP-1/TIMP-1 ratio, which were measured after two and four weeks of treatment.

\subsection{Data analysis}

We used SPSS 20 for Windows. Statistical analysis used ANOVA and least significant difference test with a level of significance of $<$ 0.05 .

\section{Results and discussions}

There were 24 subjects that met the inclusion criteria of this study. Two subjects died from the $\mathrm{K} 1$ and $\mathrm{K} 2$ group. The mean initial body weight of the $\mathrm{K} 1$ group was $303.83 \pm 37.46$ grams, with a final body weight of $294.00 \pm$ 39.81 grams. The mean initial body weight of the E1 group was $312.50 \pm 62.40$ grams, with a final body weight of $318.50 \pm 66.46$ grams. The mean initial body weight of the K2 group was $319.00 \pm 26.57$ grams, with a final body weight of $294.40 \pm 53.23$ grams. The mean initial body weight of the E2 group was $300.33 \pm 18.55$ grams, with a final body weight of $275.33 \pm$ 33.57 grams.

The results of this study showed that the mean systolic and diastolic pressure of the pulmonary artery in the group of pomegranate extract-treated mice was observed to be lower 
than that of the control group after two weeks or four weeks $(p=0.002)$. The mean pulmonary artery pressure of the group treated with pomegranate extracts was lower than that of control model mice, significantly different $(\mathrm{p}=$ 0.001 ) in the observation after two weeks or four weeks. This suggests that the administration of pomegranate extracts may prevent pulmonary artery pressure increase and inhibit $\mathrm{PAH}$ progress.

The mean systolic and diastolic pressure of Sprague-Dawley mice with similar age and weight in the preliminary study was $45 \mathrm{mmHg}$ and $19 \mathrm{mmHg}$, respectively. The mean systolic pressure of PAH model mice in a previous study was $80 \mathrm{mmHg}$, and the mean systolic pulmonary artery pressure of normal control mice was 30 mmHg (Bogart et al., 2009). A lower mean pulmonary artery pressure in the pomegranate extract group proved that these extracts may decrease pulmonary artery pressure in monocrotaline-induced PAH model mice. The pulmonary arterial pressure of monocrotaline PAH model mice treated with pomegranate extracts significantly decreased compared to that of the control group (Hanif, 2010).

Figure 1 described about the comparison the number of cells expressing MMP-1 between treatment and control groups after two and four weeks. The number of cells expressing MMP-1 in the right ventricular heart muscle of the control mice was less than that of the group given pomegranate extracts after two weeks or four weeks $(\mathrm{p}<0.001$ and $\mathrm{p}<0.001$, respectively).

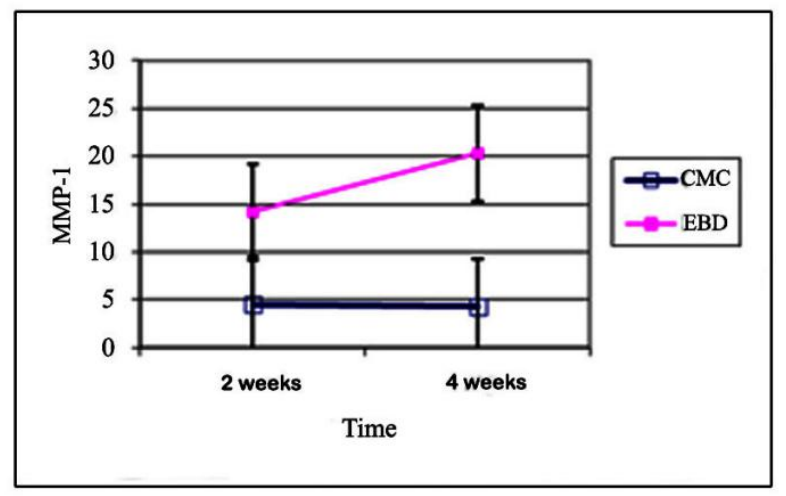

Figure 1. MMP-1 expression
The average number of cells that expressed MMP-1 in PAH model mice that received pomegranate extracts increased compared with PAH model mice not given pomegranate extracts at two weeks and four weeks of observation. Transgenic expression of human MMP-1 decreases smooth muscle cell proliferation and inhibits the excessive accumulation of collagen in the pulmonary artery. Upregulation of MMP-1 may decrease severe PAH and open the possibility of therapeutic intervention.

Administration of pomegranate extracts in the liver fibrosis model mice led to an increase in MMP-1 expression (Yuniarti, 2012).

While the number of cells expressing TIMP1 was explained in figure 2 . On the contrary with MMP-1, the number of cells expressing TIMP-1 actually was more apparent in the control group mice than in the treatment group, which was given pomegranate extracts for two weeks and four weeks of observation $(p<0.001)$.

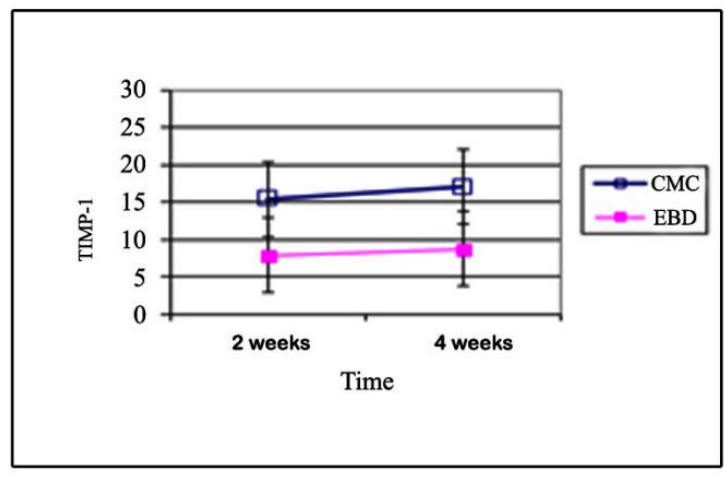

Figure 2. TIMP-1 expression

In figure 3 , the ratio of cell numbers expressing MMP-1/TIMP-1 was higher in the group of mice treated with pomegranate extracts than in the control group mice and significantly different in observations after two weeks or four weeks $(p<0.001)$. The TIMP-1 expression rate of the treatment group mice decreased and is significantly different from that of the control group. Expression of TIMP-1 increased again in the control group mice as well as the treatment group mice after four weeks but did not differ significantly between two weeks and four weeks. This proves that there are obstacles to 
MMP-1 activity that can cause the accumulation of MES protein and right ventricular heart muscle fibrosis. This is in line with other studies that considered the use of pomegranate extracts as an adjunctive therapy to the existing standard therapy to decrease PAH-related morbidity and mortality.

The increased MMP-1/TIMP-1 ratio after pomegranate extract admission can mean that there is an increase in MMP-1 activity and reduced resistance to MMP-1 activity. MMP-1 more actively degrades the MES protein so that right ventricular heart muscle fibrosis can be inhibited or reduced. The ratio remained significantly increased after two weeks and four weeks of pomegranate extracts. The increased MMP-1/TIMP-1 ratio in this study is due to increased MMP-1 expression and decreased TIMP-1 expression. Another study also found an increase in the ratio of MMP-1/TIMP-1 in the tunica adventitia pulmonary vein model of PAH experimental animal (Rahman, 2016). The MMP-1/TIMP-1 ratio under normal circumstances is $1: 1$. Increased ratios led to increased MMP-1 activity and increased MES protein degradation including collagen degradation.

There was a significant decrease in the number of TIMP-1 expression cells in the tunica adventitia of the pulmonary vein models of the PAH-induced treatment group mice compared with control group mice (Rahman, 2016).

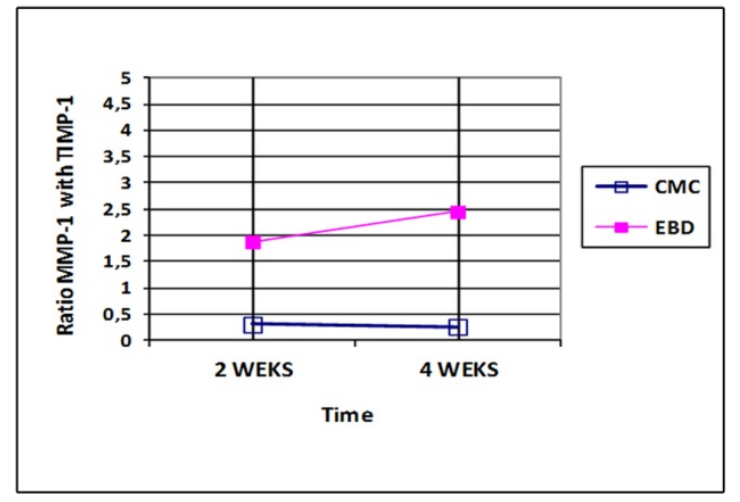

Figure 3. The ratio of the number of cells expressing MMP-1 / TIMP-1

Figure 4 showed that the number of cells expressing type 1 collagen in the treatment group mice was no different than in the control group mice after two weeks of observation $(\mathrm{p}=$ 0.193).

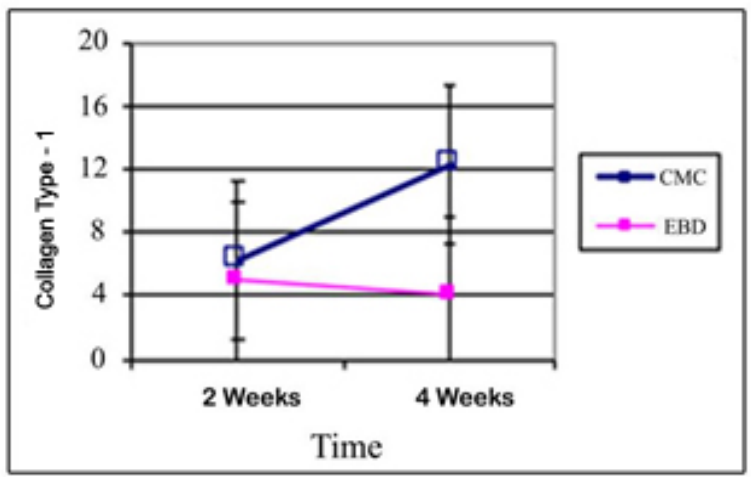

Figure 4. Expression of collagen type-1

In contrast, the number of cells expressing type I collagen in the group of mice treated with pomegranate extracts was much lower than in the control group mice after four weeks of observation and was significantly different $(\mathrm{p}<$ 0.001 ). Pomegranate extracts have the ability to inhibit the increase of the number of cells expressing type I collagen of the right ventricular heart muscle. The number of cells expressing type 1 collagen in the four-week control group was more statistically different than in the two-week control group. These results are consistent with other studies showing a decrease in the number of cell expression of type I collagen in the pulmonary vein of the monocrotaline-induced PAH in treatment group mice (Rahman, 2016). The effect of type I collagen decrease is related to the active ingredient ellagic acid and polyphenols in pomegranate extracts. These results suggest that pomegranate extract administration may inhibit PAH progression, but more research is needed to evaluate the effectiveness of the long-term use of PAH progression.

\section{Conclusions}

The effectiveness of pomegranate extracts in increasing MMP-1 levels, lowering TIMP-1 levels, increasing MMP-1/TIMP-1 ratio, and decreasing type I collagen in two weeks and four weeks of observation showed that pomegranate extracts may inhibit the initial fibrogenesis process while increasing fibrosis resolution. 
Pomegranate extracts are expected to prevent fibrosis and reduce the degree of fibrosis that has already occurred. The results of this study are expected to be a consideration for the use of pomegranate extracts as an adjunctive therapy to existing standard therapy to decrease PAHrelated morbidity and mortality.

\section{References}

Bogaard, H.J., Natarajan, R., Henderson, S.C., Long, C.S., Kraskauskas, D., Smithson, L., Ockaili, R., McCord, J.M., Voelkel, N.F. (2009). Chronic pulmonary artery pressure, elevation is insufficient to explain right heart failure. Circulation, 120, 1951-1960.

Haber, S.L., Joy, J.K., Largent, R. (2011). Antioxidant and antiatherogenic effects of pomegranate. Am J Health Syst Pharm, 68:1302-5.

Hanif, K. (2010). Effect of aqueous extract of Punica granatum (Pomegranate) on monocrotaline-induced pulmonary hypertension in rats. Recent trends and future perspectives in high altitude pulmonary research. Abstracts, Leh Ladakh J\&K India. September 27-30.

Jeffery, T.K., Morrell, N.W. (2002). Molecular and cellular basis of pulmonary vascular remodeling in pulmonary hypertension. Progress in Cardiovascular Diseases, 45, 173-202.

Jurenka, J. (2008). Therapeutic applications of pomegranate (Punica granatum L). A review. Alternative Medicine Review, 13, 128-44.

Mandal, A. (2014). Recent advances in understanding the role of matrix metalloproteinases in pulmonary arterial hypertension: A review. International Journal of Basic \& Applied Physiology, 3, 1-23.

Rahman, M.A. (2016). Effect of standardized ellagic acid $40 \%$ pomegranate extract on progressivity resistance of arterial hypertension [dissertation]. Surabaya : Airlangga University.

Vidal, A., Fallarero, A., Pena, B.R., Em, M., Gra, B., Rivera, F. (2003). Studies on the toxicity of Punica granatum L. (Punicaceae) whole fruit extracts. J Ethnopharmacol, 89, 295-300.

Wardle, A., Tulloh, R. (2012). Update on pulmonary arterial hypertension in children: Management strategies and clinical utility of Sildenafil. Pediatric Health Med Ther, 3, 59-67.

Yuniarti, W.M. (2012). Antifibrotic effects of pomegranate extract (Punika granatum L) in liver fibrosis. [dissertation]. Surabaya: Airangga University.

\section{Acknowledgment}

We want to express our gratitude to our seniors in the Division of Cardiology, Department of Pediatrics, Dr. Soetomo General Hospital Surabaya. We would also like to thank Dr. Rizqi Rokhmadhoni P., SpA, and Dian Asri for their help in editing this manuscript. 